Choreographed Soft Morphologies: Exploring New Ways of Ideating Soft Architecture Through Material Elasticity
KEYWORDS

Choreography; embodied interaction; choreography; soft interface; elasticity; architecture; motion capture technology; physical-digital.

HOW TO CITE

Castán, Marina and Daniel Suárez. 2018. "Choreographed Soft Morphologies: exploring new ways of ideating soft architecture through material elasticity".

Temes de disseny 34: 60-73

CASE STUDY
This research aims to contribute to the current field of architectural design by offering evidence of how a collaborative and embodied approach to soft architecture can inform a new physical-digital design process. Current design technologies (e.g. sensors, 3D scanners, procedural modelling software), together with the use of the body as a source for designing a space, offer new methods and tools for designing architecture (Hirschberg, Sayegh, Frühwirth and Zedlacher 2006). However, the potential for experiencing and digitally capturing a soft and elastic material interaction through the body as a dynamic system capable of informing soft architectural design has not yet been widely explored. By using the felt experience as a tool for design, we allow the material to express its qualities when activated by the body, revealing its form instead of it being imposed from outside (DeLanda 2015). Taking an embodied approach used in interaction design and fashion design (Loke and Robertson 2011; Wilde, Vallgårda, and Tomico 2017), this research proposes a hybrid method to explore a textile-body ontology as an entity that has the potential to design a space, along with the use of motion capture technology in an effort to re-connect the experiential (the body) with the architecture (the space).

Through a custom-made interface, made of soft and hard materials, we explored the dynamic and spatial qualities of material elasticity through choreographed body movements. The interface acts as a deformable space that can be shaped by the body, producing a collection of form expressions, ranging from subtle surface modifications to more prominent deformations. Such form-giving processes were captured in real time by three Kinect sensors, offering a distinct digital raw material that can be conveniently manipulated and translated into architectural simulations, validating the method as a new way to inform soft architectural design processes.

The findings showed that: 1) the direct experience of collaboratively interacting with a soft and elastic interface allows the identification of the dynamic qualities of the material in relation to oneself and others, facilitating an immediate spatial meaning-making process; 2) exploring the design of a soft and elastic space through choreography and motion capture technology contributes to the creation of augmented relational scales across physical and digital M. CASTÁN / D. SUÁREZ 
realms, proposing a new hybrid design method; 3) the soft and elastic interface becomes a new entity when shaped by the body (textile-body ontology) giving the opportunity for a variety of formal expressions and offering a source of digital raw material for architectural design.

$$
\stackrel{1}{\text { INTRODUCTION }}
$$

In the same spirit, this research aims to engage with architecture in a more experiential way, connecting physical spatial experiences with digital design processes. Our contribution lies in the creation of a hybrid method Our contribution lies in the creation of a hybrid metho The use of the body as a choreographic tool to inform the that simultaneously connects physical actions with their design of space has been widely explored by architects in digital counterparts. By doing this, various stakeholder recent decades. Architects have collaborated with dancers can co-shape the space and, subsequently, the architectura and choreographers to translate movement into notation form in both the physical and the digital realm. The even systems through analogue-digital workflows. In this way, is captured in real time and enables the digital creation of the design of an architectural form or spatial arrangement visualisations, offering a new soft form vocabulary, based is often materialised through rigid and static prototypes on the particularities of the temporal transformations of the (Kato and Glynn 2017; Salazar Sutil 2015). Pacher's work textile-body ontology. In this way, the direct experience of takes a performative approach to architecture and proposes inhabiting a space that is soft and dynamic would not only working with our sense of movement and its ability to affect inform new spatial logics but would also offer a catalogue Using Kinect sensors, Pacher developed a mobile applica- tion between the material and the body in movement. The tion that allows the user to use his or her actions as inputs digital captures are first re-constructed as a mesh (to make to influence the design of space in real time (Pacher 2014). them stable enough to work with modelling software) and Architect and dancer Chryssa Varna uses dance notations later manipulated to display their potential as architectura to create a choreography for designing kinetic architecture design material.

based on the use of robots and paper fans as responsive devices, offering a new spatial design language (Varna 2013). bodied design result of using a combined perspective of emdesign (by means of choreography) and technology tile architecture, this research explored a collaborative (by means of motion capture tools and modelling software) process of soft architecture that goes beyond the design of od brings allows the exploration of the qualities of a so an architectural form, aiming to introduce a new method space and the relationship to others in a very immediate for designing soft architecture through material elasticity manner. Secondly, the digital captures enable working at at multiple scales (a surface, facade, transitional space or different scales and the generation of visuals of soft archipavilion). The design of a soft space by means of body-mate- tectural designs, providing a distinct digital material wit rial interactions has received little attention within the field numerous formal and spatial explorative possibilities. A of architecture in contrast to other fields such as interaction hybrid method such as this brings together the expertise of design or fashion design in which the relationship between architects, textile designers, dancers and choreographers in the material and the body is often more direct. The most a collaborative process that enables analysis and reflection obvious reason that emerges for this is one of scale: archi- on the outcomes from different perspectives, contributing tects' direct experience with materials is often relegated to to expanding the understanding of space towards a more a physical model that does not match the scale of the body. personalised concept thereof. We start by providing a brief Thus, the hybrid method we are putting forward in this introduction to the concept of materiality and the relationresearch project would allow architects to improve their ship between matter and form from a philosophical point of interaction with the material in terms of direct experience view, continuing by offering a brief overview of embodied by bridging the gap between the scale of the model and the interaction design. Finally, we describe our experimental scale of the body. As Schillig points out, the interactive, setup and the hybrid method before analysing the value of kinetic and performative aspects that come into play when collaboratively designing soft architecture through choreapproaching the design of a space mediated by the mate- ography and motion capture technology

rial and at the scale of the body results in a dynamic and transformative space that "anticipates a precursor of virtua space - a virtual space where the body is not suppressed, but for which it becomes the initiator of space". Performative Geometries (A. Agkathidis et al. 2010) documents the way
that a collection of soft envelopes challenges the definition of that a collection of soft envelopes challenges the definition of
space traditionally understood as something static and rigid space traditionally understood as something static and rigid,
offering instead an organic and emergent space mediated offering instead an organic an
by the materiality of textiles.

TEMES DE DISSENY \#34 $\stackrel{2}{2}$ THE CONCEPT OF MATERIALITY IN THE PHYSICAL AND DIGITAL REALMS sarily requires different approaches and methodologies in CASE STUDY
Working within a physical-digital context in design neces- ontrast to other design processes that do not involve digital digital realm introduces computational material computerisation, as they believe that the majority of contemporary architecture is still besed on an approach that uses computational design as a way to operate more efficiently or faster within the design process (computerisation) rather than as a new way of thinking that generates more information and brings certain particularities (computation).

The origin of this shift in architecture from a static and linear process to a more complex and dynamic system comes from automated design and cybernetics. Approaches such as this position computers as an extension of human beings, with their proximity to human thinking (Ahlquist and Menges 2011)

This understanding of the physical and the digital world as a system is very much in line with the contemporary approach within Human-Computer Interaction Design, in which the physical and digital are understood as a composition rather than as two separate things (Wiberg and digital materials are used within the design process. In this sense, our method combines a soft and elastic interface connected to the digital realm through motion capture sensors, operating as an extension of our actions, behaving as an integrated system that unfolds its own materiality, thus allowing for a continuous design flow. Much has been said about the materiality of computational technology and the turn towards an understanding of it as an expressive and graspable material (Brownell 2006; Landin 2005; Löwgren and Stolterman 2004; Vallgårda and Redström 2007; Vallgårda and Sokolar 2010). Vallgårda argues that the computer becomes a material for design, helping designers to achieve their creations through complex form-giving processes that necessarily contain the interaction between the physical material, the computational material and the interactions carried out by the user (Vallgårda 2014). Landin claims that computational technology within the context of interaction design is not just a neutral tool to implement the technical aspects of design, but an 'expressive design material instead' (Landin 2005, 117). Acknowledging the expressive and tangible qualities of the digital material enables a more integrated creative process, in which the physical and the digital are conthuously fed into each other, not only co-shaping a particular design process but also resulting in distinct outcomes. How do we design, then, with material whose qualities are different? Since the publication of the foundational book on interaction design by Dourish (1999) in which he develops an embodied approach to interaction design, new methodologies that use, in different ways, the notion of the lived body, have emerged. This notion come from a break with the mind-body dualism proposed by Heidegger, Merleau-Ponty and other theorists such as Dewey (Pragmatist Aesthetics), Bourriaud (Relational Aesthetics) DeLanda, Braidotti or Barad (New Materialism). In the last decade, a growing interest in these theories have resulted in a variety of methods that offer an alternative to othe traditional approaches to design. Loke and Robertson give
a comprehensive account of the way that design research-

M. CASTÁN / D. SUÁREZ he importance of distinguishing between computation an

use the body as a source to inform design and analyse,

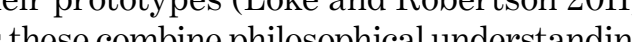
with practice-based design research using the material, the ody and the context as materials for design. Importantly digital technologies transform our realities into technologbecome central within the early phases of design (Wilde, da, and Tomico 2017).

Drawing on the notions of 'material capacities' (DeLanda 2015), we will demonstrate how a combined approach of embodied design and motion capture technology relation to soft architecture fosters a new spatial experince and contributes to the creation of a novel form-giving process for architectural design.

3

A CONTEMPORARY PHILOSOPHICAL CONTEXT OF ARCHITECTURE

DeLanda states that there is a conceptual shift regarding materials as systems that are defined not only by their propview, also by their capacities. From a philosophical point DeLanda terms it (DeLanda 2015, 85:2), reveal a different

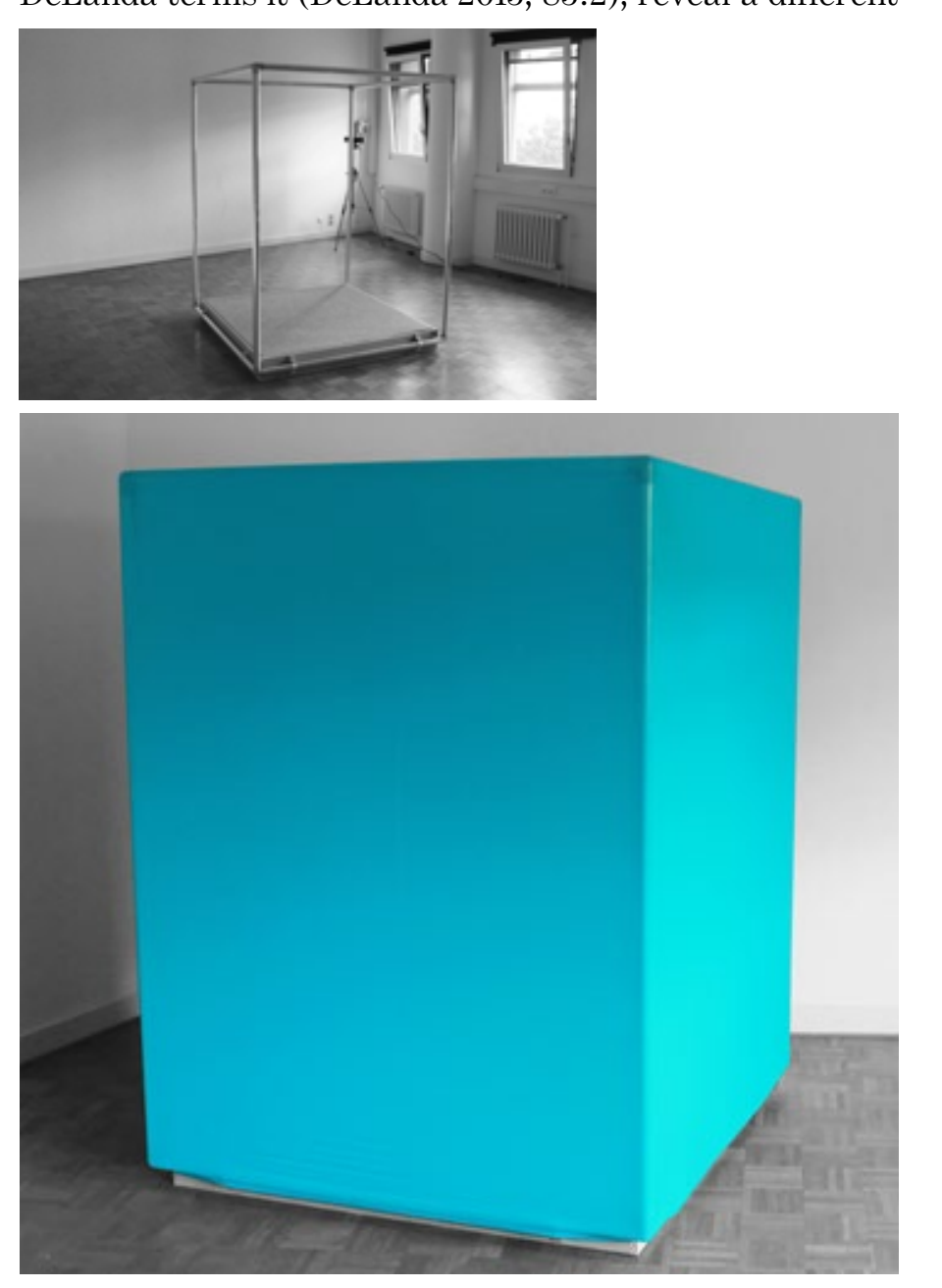




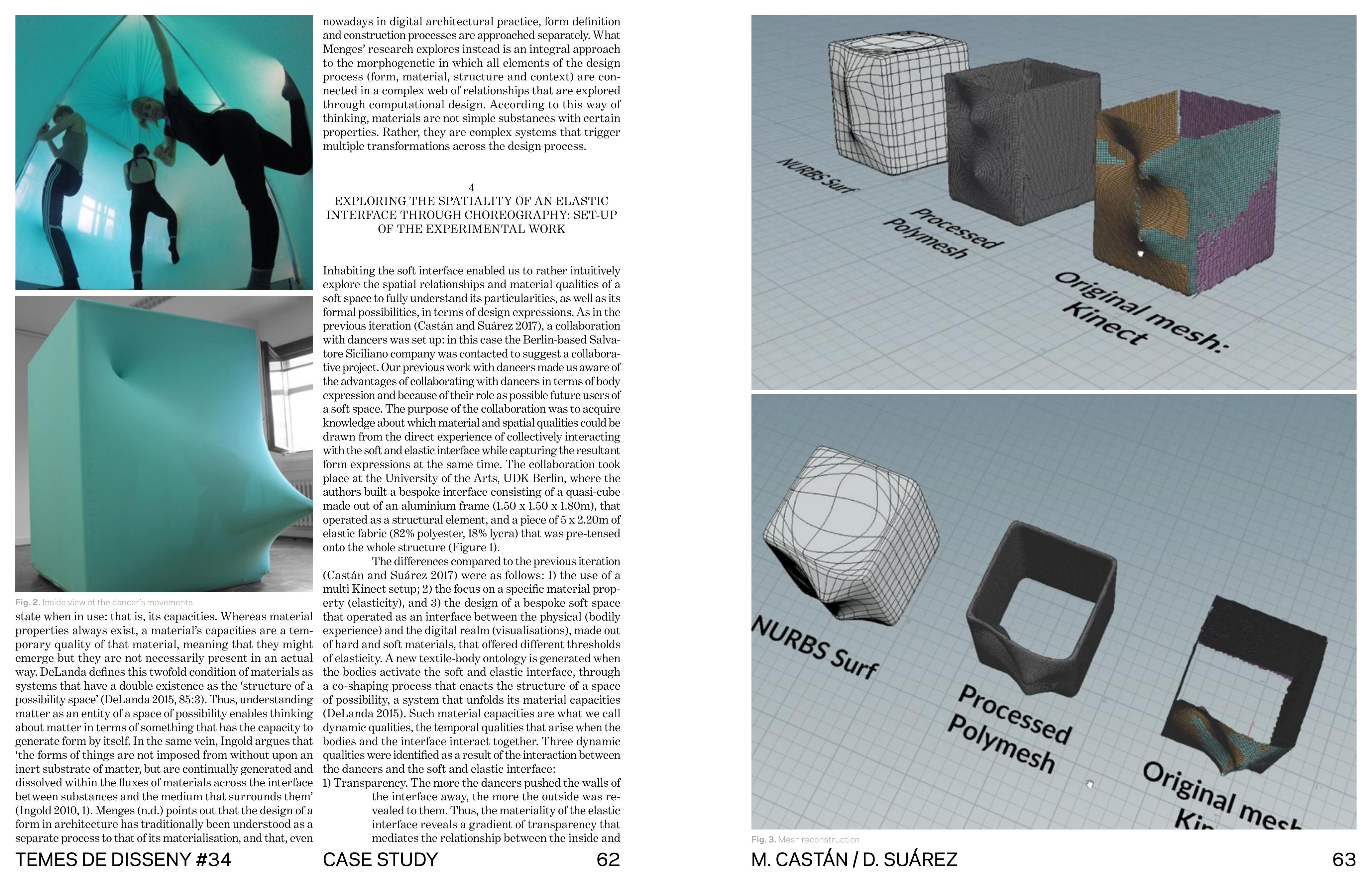




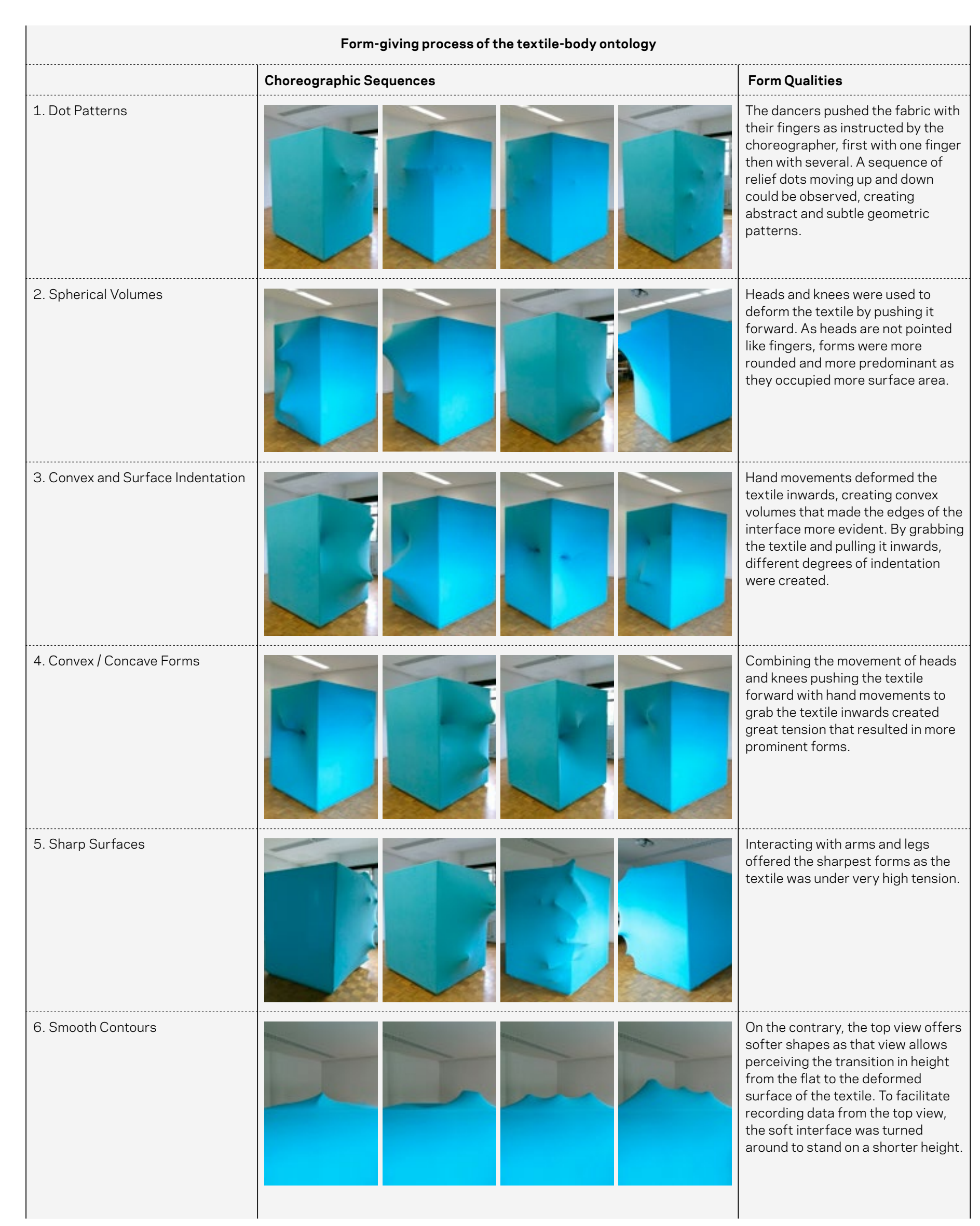

Table 1. Form-Giving Procese

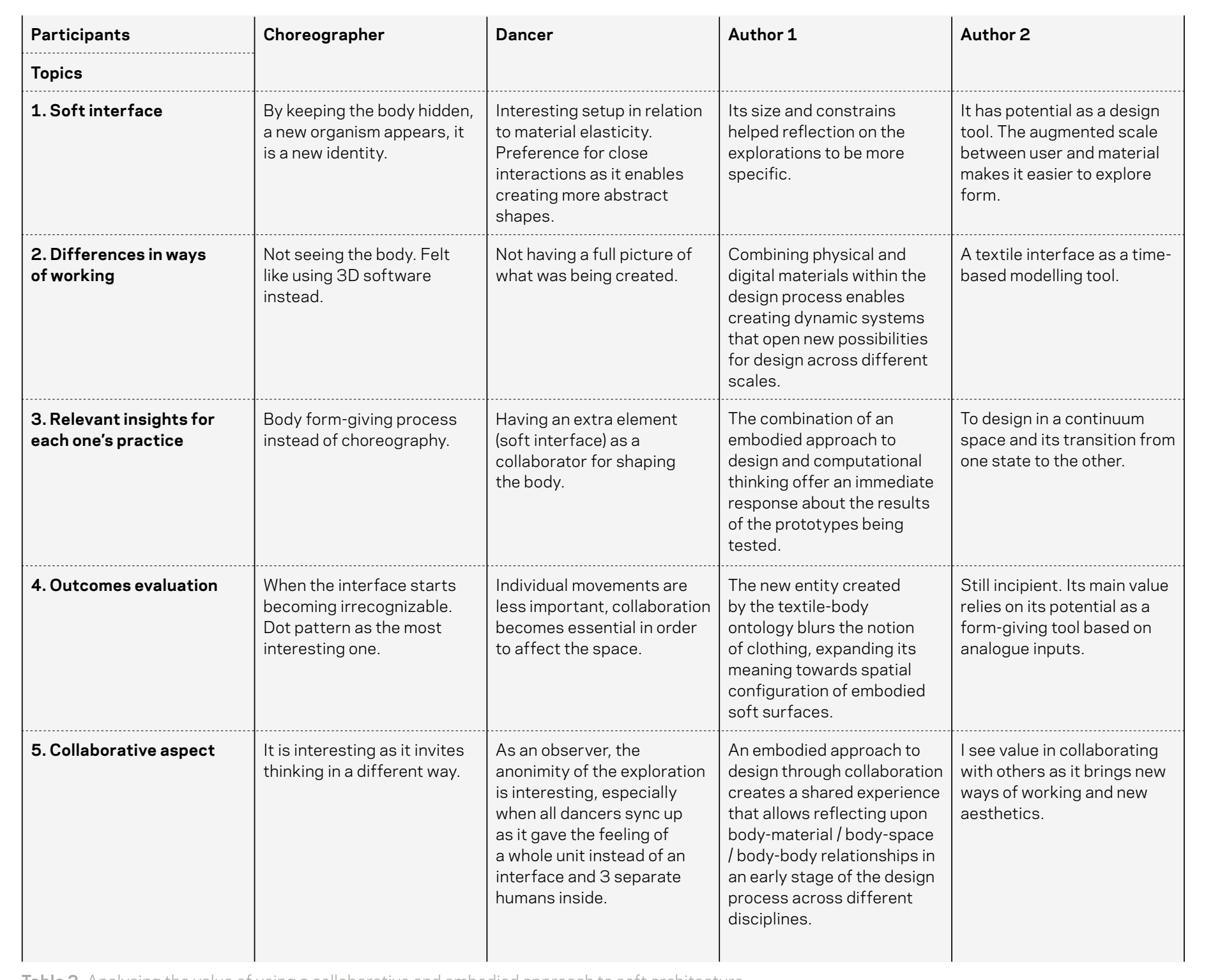


the outside, creating dynamic interactions between ing the captures that are presented here, they turned into users and space. $\quad$ unexpected forms and deformations of the soft and elastic volumes and forms, offering very defined shapes three Kinect sensors, a raw mesh is created, allowing the when the body activates the soft interface. Its ad- designer to re-construct it and project different condition ptability allows users to molify the space aceord- and eriteria to obtain specific outputs (Figure 3 ). ing to their needs, taking up room when needed or using the minimum space otherwise.

The forms created by the textile-body ontology hold certain qualities of plasticity and dynamism that make The soft interface operates as a pre-tensed envelope, allowing users to experience how it feels sions, in blows an understanding of the singularities of a soft performing counterbalanced movements through and elastic space and its expressive qualities as a system, as different thresholds of elasticity. a new ontology that generates a distinct form-giving process. The behaviour of the soft interface is determined

As a result of the co-shaping process, a collection by the combination of the fabric material properties and the of morphologies was created. Table 1 shows a selection of restrictions imposed by the aluminium frame, thus in an the choreographic sequences performed by the dancers, as elastic yet stable combination. Subsequently, the capacity to well as a brief description of the resultant form expressions. be stretched out by the body creates a volume that results in The dancers, in dialogue with the choreographer, performed a specific form, in a temporary event that modifies one side six different choreographic sequences using different parts of the interface from being a plane to becoming a three-diof their bodies, indicated in the description of each of the mensional surface. The data captured by the Kinect sensors form expressions: 1. Dot Patterns, 2. Spherical Volumes, 3. changes over time, introducing a 4D aspect that makes this Convex and Surface Indentation, 4. Convex/Concave Forms, data significantly different from the one that results from a 5. Sharp Surfaces, 6. Smooth Contours. static body, creating a temporal aspect and generating data The dancers started by first performing a more that updates constantly. Moreover, by examining the data localised movement with their fingers, continuing by grad- with the procedural modelling software, hidden spaces that ually integrating other parts of their bodies to have a more are not visible within the physical realm can be discovered, significant impact on the walls of the elastic interface. In- as the Kinect sensors reconstruct some of the parts that tuitively, they began to help each other to gain more control are not visible, creating new possibilities of spatial configin deforming the walls. As Figure 2 shows, dancers made uration. Within a soft architecture context, different ideas use of the rigid frame to hold themselves when stretching of potential applications emerged, such as, for example, the the walls. A GoPro camera was placed inside the elastic idea of a soft space inside a built space, a soft wall or soft cube, and a video-recording camera outside of it in order to window that could mediate as an interstitial space between capture the dancers movements and document both inside the facade and the interior of a building, modifying natural Three Kinect sensors were triangulated to get a the textile and pushing it outwards: a similar concept to 60-degree capture of the explorations. Brekel multi-Kinect the recently built Guelmim airport, in Morocco, by Groupe software was used to record raw data retrieved from the 3 architects. They wrapped the building with a metal mesh Kinect cameras which were later imported into SideFX skin, creating a light-filtering facade, mediating the relaHoudini software to join the three different meshes of each tionship between inside and outside. The results obtained position into one single object and perform a subsequent re- from our elastic interface could drive similar operations. construction of this joined mesh. Once the mesh was cleaned, Figure 4 shows how the dynamic quality of transparency the still frame geometry was exported as a *obj file, and is translated into a colour gradient map and applied to the imported in Rhino3D to manipulate it within a Grasshopper reconstructed mesh. In this way, some graded distributed environment.

areas of transparency are created, making it possible to The improvements in the physical-digital setup evaluate the distances between the face centres of an initia mentioned above responded to the following: the need to state of the mesh and the manipulated one

obtain a smaller amount of data that was more precise, a

physical space with certain constraints (related to dimen-

sions and material properties) that allows the participant

to be more specific in relation to the findings, and finally

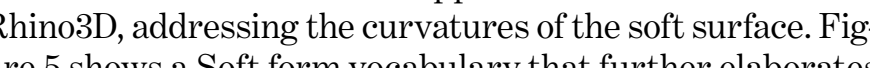
(bisualisation of a speculative spaces, in this case, triggered by the elas tic property of the urban scenario in which certain parts of a building's ski material (a matter-form relationship). The data captured by the Kinect sensors enables different perspectives on the soft elastic interface, as Figure 3 shows. By handling dat that is based on transformation processes, certain random events emerge, such a glitch, that in the context of computing refers to a malfunction in a programme or interface; some-

TEMES DE DISSENY \#34
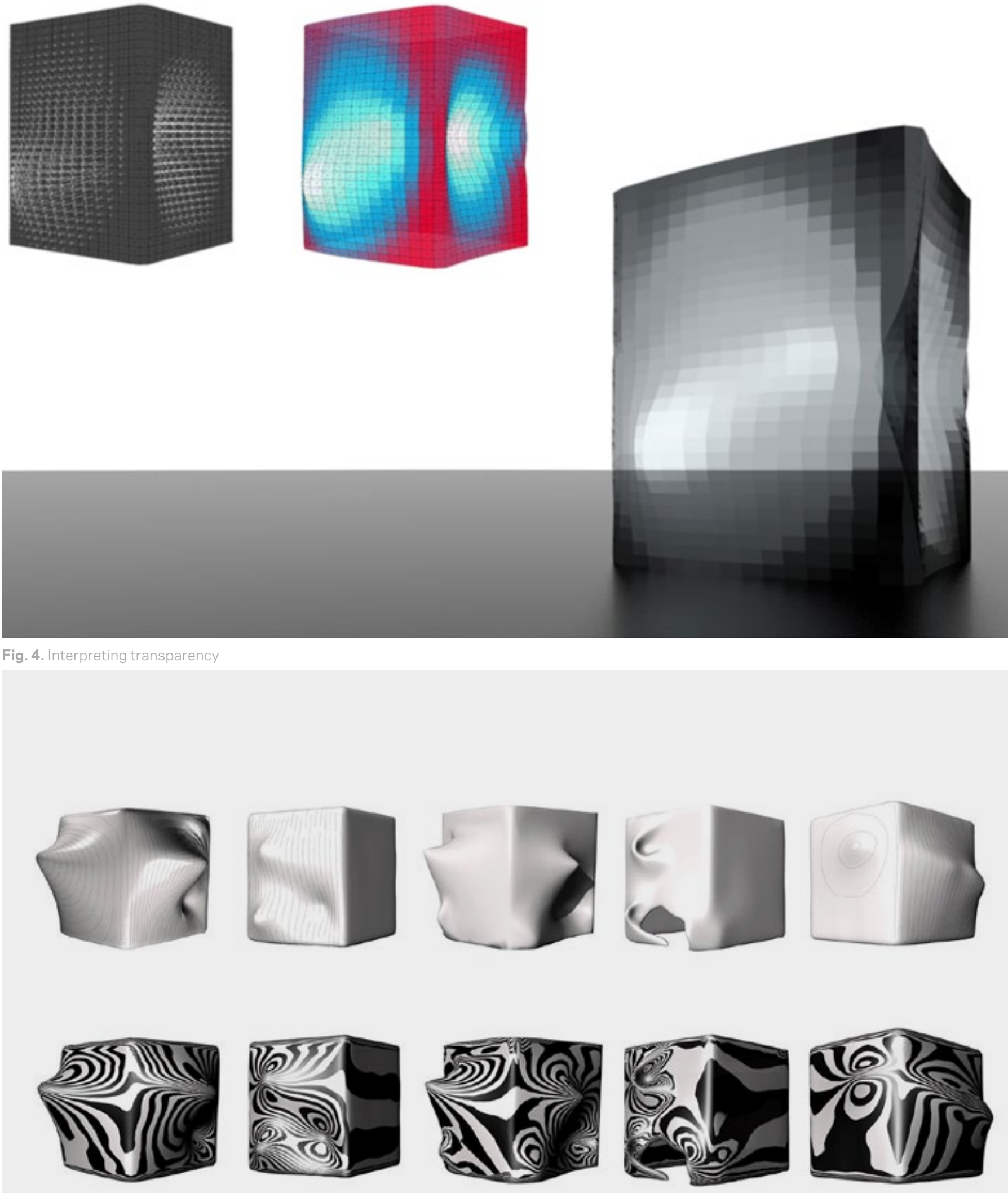
urban scenario in which certain parts of 
5

ANALYSING THE VALUE OF

DESIGNING SOFT ARCHITECTURE THROUGH EMBODIED INTERACTION AND MOTION

CAPTURE TECHNOLOGY

In order to assess the value of the collaboration, an open coding process used in Grounded Theory (Flynn and Korcuska 2018) was used to analyse and identify com

Both authors, the choreographer and one of the ancers answered a brief questionnaire to extract the most interesting aspects from the collaboration. The questionnaire focuses on five topics: 1) The design of the soft interface 2) Differences in ways of working 3) Relevant insights for each participant's practice 4) Outcomes evaluation, and 5) The collaborative aspect.

\section{The soft interface}

What do you think of the soft interface?

For the choreographer, this exploration connects with his idea of architectural dance, as it is a very architectural way of composing with the body. 'By keeping the bodies hidden, a complete organism appears, it is a new identity'. Dancers and the soft interface together defined a new body, creating architectures of movement in the space.

The dancer found the set-up interesting and enjoyed interacting with the elastic properties of the material. She preferred the interactions that happen closer to each other: 'I felt we were able to create more shapes that were termined how much the dancers could affect its shape. Author 1 found that the dimensions and the for of the soft interface helped to narrow down the scope of the esearch and therefore enabled more specificity in the results and the findings. 'The idea of focusing on a bespoke space as a module facilitates the process of digitally speculating on a more complex application by grouping together different terms of how the interface could be improved, by increasing the numbers of soft interfaces and dancers, more complex spatial configurations would be created.

Author 2 sees potential in the soft interface as a design tool: 'in this augmented relational scale between use' and material it is easier to explore and to sculpt form while one experiences in the first person how material behaves'.

2. Differences in ways of working

What did you find different from your usual way of working?

According to the choreographer, movement was mainly led by the soft interface as he tried to achieve what he had in mind, but the interface behaved quite differently from how he expected it to, so he decided to improvise by relying upon the immediate outputs. During the explorations he felt that 'the dancers and the interface defined a new body that creates architecture of movements in the space. In his opinion, an invisible machine with different

TEMES DE DISSENY \#34 is one object rather than three separate humans inside'. physical hypothesis to reflect upon the relationship betwe value of this toolset is as a form-generation or form-giving

3. Relevant insights for each participant's practice What did you find interesting/useful from your perspective?

The choreographer found it quite challenging to create movement with the constraints of the soft interface, s he could not see the dancer's body: 'You can't think of . However, he thought it was inspiring as a way to get into aphy from a different point of view.

For the dancer, the interface operated as a sort of shield. 'I was no longer visually exposed, so my movement the way I looked were not that important any more'. In ther to have more impact on the surface of the interface, ey intuitively started to help one another by acting as -balances.

What Author 1 found most interesting was the fact that by working motion capture technology, the variety of possibilities was very exciting in terms of the digital visuallsations and speculation that this way of working offers. Both an embodied approach to design and motion capture technology offer an immediate response to the results of the prototypes being tested.

Author 2 sees the potential in 'designing a continuum space and having the opportunity to explore in the computer not only interesting forms but also transition from one state to another'.

\section{Outcomes evaluation}

What is your opinion regarding the outcomes of the exploration?

The choreographer found it particularly interesting that 'the interface becomes unrecognizable as the dancers deform the corners of it', and that by adding more dot patterns were most interesting and elegant.

\section{CASE STUDY} based on body instructions which brings a very interesting

In the next section, a brief analysis of the main offers a more precise picture of the main feedback from each

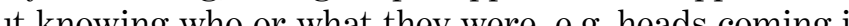
and out of the interface. For her the patterns generated by the synchronized movements of each dancer's fingers were the most interesting as 'it gives a feeling that the interface Author 1 thinks that both the physical and the digital results are relevant in the sense that they work as a body and spaces in terms of experience and the relationship between form and matter. From the point of view of the textile design, 'the image of several bodies interacting in side an elastic space blurs the notion of clothing expanding its meaning towards a spatial configuration of bodies and soft surfaces in which one becomes the other, making them inseparable'.

Author 2 believes that they are still at an early stage, but that they present many possibilities through the variety of parameters that can be modified according to specific design task. Potential translations into architecture include the structural performance of a particular material its qualities or other rendered properties. 'The principal process similar to a sort of explicit modelling tool, but in this case mediated by analogue inputs'.

ographer and the dancer, not seeing each other while performing forced them to find other ways of (hay the dancers interacted with each other.

b) The choreographer (Topic 1), the dancer (Topic 5) and Author 1 (Topic 4) coincide in their opinion that the soft interface, when interacting with the bodies, becomes a whole new entity.

Whilst the choreographer and the dancer identified this new entity by relating to it through body and movement, Author 1 observed that by seeing it as an extension of clothing, a transitional state between clothing and architecture creates a new ontology. c) The choreographer (Topics 2 and 3 ) and Author 2 (Topics
1 and 2) agreed on the potential of the soft interface as a body form-giving process.

Interestingly, both the choreographer and Author 2 saw the interactions between the soft interface and the dancers as a 3D modelling tool.

Author 1 (Topic 2) and Author 2 (Topic 2 and 3) consider the particularities of the physical-digital set-up especially relevant as a tool that opens up new design possibilities.

For Author 1, the physical and digital set-up brings a new design space for exploring the materiality of textiles on different scales.

What do you think about the collaborative aspect of the project?

The choreographer considers the collaboration as an 'invitation to think differently, e.g. to imagine a wall aesthetic. You can arrive at the conclusion of having a wall with dots'. The dancer noted that 'working with an interface that manipulates the shape of the body' was interesting to her, as dance is related very much to the shape of the body. She also mentioned that the collaborative aspect also affected their understanding of the space, as it became more relevan by being defined by the interface.

Author 1 considers the collaborative aspect to be essential when it comes to bridging two disciplines, and that in the case under discussion in this paper, 'the collaborative aspect becomes not only a co-shape process involving different stakeholders, but also the material.

Author 2 sees value in collaborating with othe disciplines, as 'different perspectives and ways of working convey in a specific research motivation, contribut

creating new ways of working and new aesthetics' findings based on participants' feedback is provided. Table 2 participant in order to compare
points in relation to the topics.

The following commonalities were identified:

the fact of to not seeing the body/outside challenging, as their

interaction was mediated by the soft interface

M. CASTÁN / D. SUÁREZ e) All participants perceived that there is value in working across different disciplines as it provides a different way of thinking about one's own practice, as
well as news methods and aesthetics (Topic 5 and Topic 3, dancer)

CHOREOGRAPHED MORPHOLOGIES SOFT ARCHITECTURE

he motivation for this research was to explore other ways designing soft architecture based on direct experience the material through embodied interaction and motion

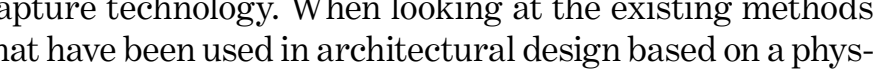
al-digital set-up, the majority of them use the body as an terface to produce a form or interactive spatial experiences, often using static and rigid materials. Thus, they do not embodied interaction as a system that is able to inform the design of space. Consequently, knowledge relating to the spacase study, this research has demonstrated how an embodied and spatial knowledge at different levels (body-material, maerial-space and body-body) across disciplines through a coldesigners and architects. The property of the material that this research explored is that of elasticity. The findings show tial experience of a soft space is somewhat limited. Through a androach to soft architecture contributes to gaining material 
1) The combined approach of choreography and motion capture technology offers a co-shaping process in
which space is shaped by the direct experience with the material, both in the physical and the digital realm.

OPEN ACCESS

This case studd is distributed under the terms of the Creative Commons Attribution 4.0

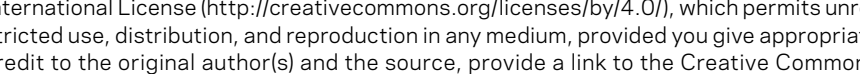

2) The use of a physical-digital method offers a set of physical and digital morphologies (the form-giving process), enabling

3) The use of an embodied approach offers the opportunity to grasp the dynamic and spatial qualities of the body-material interactions. It enables an immediate understanding of the materiality of the textile with regard to the creation of space (inside-outside) and the relationship to others that share the same space (the meaning-making process).

4) Working collaboratively enables new perspectives to be brought from other research fields. In the case presented in this paper, working with dancers as experts on body movement contributed to the generation of more precise outcomes of the form expressions as well as feedback from first-hand experience (possible future users).

Choreographed morphologies aimed to offer a new method for designing soft architecture by re-connecting the body with space by means of an embodied experience. Although the outcomes of the method presented in this paper are not definitive, they prove the method's potential as a way to open up new design processes and new ways of interacting within a soft space. It brings a new perspective on how the dynamic qualities of a soft and elastic material can be used to inform the early stages of an architectural design process. Thus, the resultant space would also affect the way users interact within the space and with others. It would allow them to have a more personalised spatial experence, enabling them to modify parts of the walls, windows, facades or other architectural elements according to their needs or motivations

Involving a larger group of participants, and using arger hard and soft material systems, would allow the project to grow in scale and to further elaborate the body-material interaction in both the physical and the digital realms. Including architects, dancers, embodied interaction design experts, textile designers and users during the design process would enrich the insights into designing soft architecture ar open up a co-design process for soft architecture design.

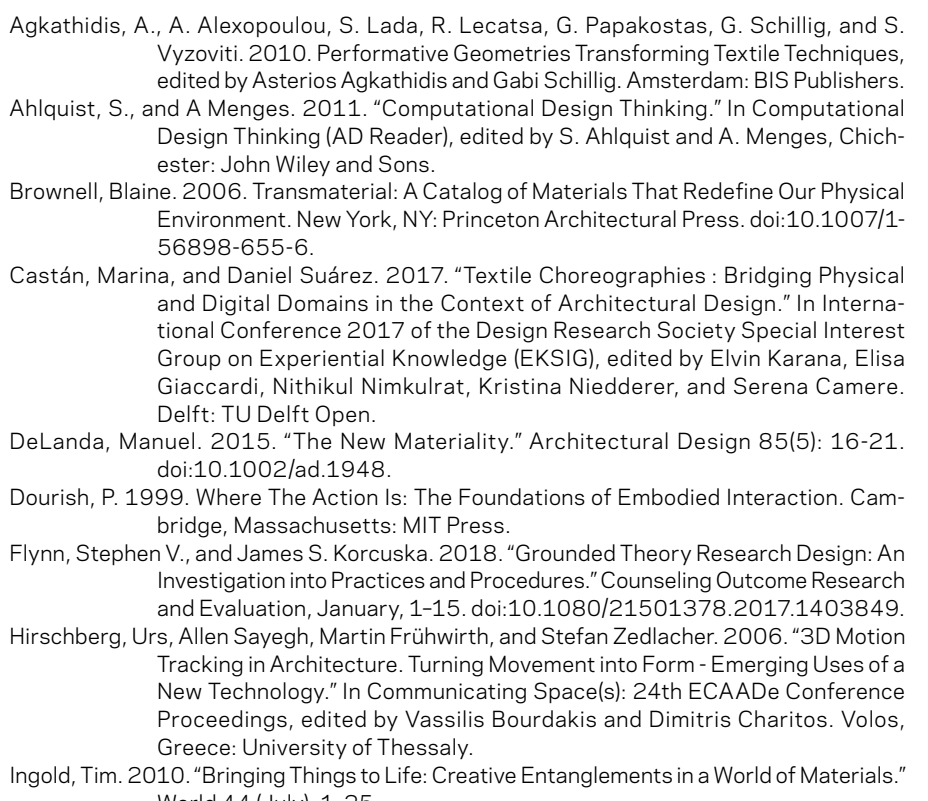
Inola, Th. 2010. "Bringing Things to the

Glynn. 2017 "Fabricating Performance: Reciprocal Constructs Dance Notation Cesign Material." "I Proceedings of the 4 th Decennial Conference on Critical USA:ACM. doi:10.1145/1094562.1094578.
Toni Robertson. 2011. "The Lived Body in Design: Mapping the Terrain

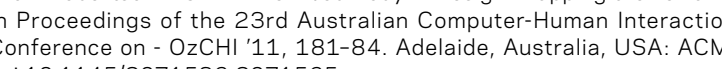
doi:10.1145/2071536.207156-8 Menges, Achim. n.d. "Design Research Agendada." "Accessed February 22, 2018. http://

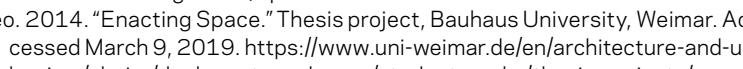

a
ment. Cambridge, Massachusetts: MIT Press.

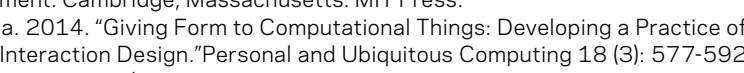
and Johan Redström. 20077. "Computational Composites." I I Proceedings of the SIICHI Conference on Humann aactors in Computing Systems
San Jose, California, 28 April - 3 May, 513-22. New York, NY: ACM

Vallgårda, Anna, and Tomas Sokolar. 2010. "Material Computing: Computing Materials" pers on Ubiquitous Computing - Adjunnct, 383-84. New York, NY: ACM 2013. "Improvisational Choreography as a Design Language for Spatia
20.1.

Cornwall: : Fascinate Conference.

Landin, Hanna. 2005." Fragile and Magical: Materiality of Computational Technology As 